\title{
Synthesis and Biological Evaluation of Novel
}

\section{Phenylcarbazoles as Potential Anticancer Agents}

Sylvain Routier, ${ }^{a *}$ Jean-Yves Mérour, ${ }^{a}$ Nathalie Dias, ${ }^{b}$ Amélie Lansiaux, ${ }^{b}$ Christian Bailly, ${ }^{b}$ Olivier Lozach ${ }^{c}$ and Laurent Meijer ${ }^{c}$

a Institut de Chimie Organique et Analytique, UMR CNRS 6005, Université d'Orléans, Rue de Chartres,

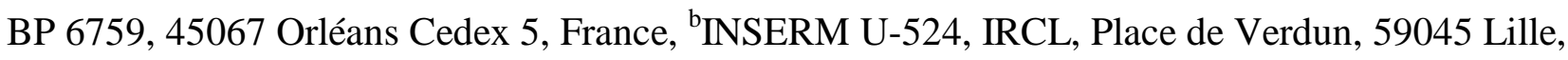
France, ${ }^{c}$ Station Biologique, place G. Teissier, B.P. 74, 29682 ROSCOFF Cedex, France

\section{Table of contents :}

Elemental analysis Table of related compounds

Routine spectroscopic data.

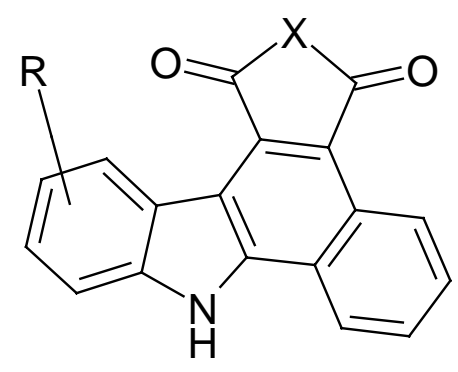

corresponding author : sylvain.routier@univ-orleans.fr 
Elemental analysis Table.

\begin{tabular}{|l|l|l|l|}
\hline Compound \# & Anal. & Calculated, $\mathrm{C}, \mathrm{H}, \mathrm{N}$ & Found, $\mathrm{C}, \mathrm{H}, \mathrm{N}$ \\
\hline $\mathbf{3}$ & $\mathrm{C}_{26} \mathrm{H}_{20} \mathrm{~N}_{2} \mathrm{O}_{5} \mathrm{~S}$ & $66.09,4.27,5.93$. & $65.72,4.41,6.04$. \\
\hline $\mathbf{4}$ & $\mathrm{C}_{25} \mathrm{H}_{18} \mathrm{~N}_{2} \mathrm{O}_{5} \mathrm{~S}$ & $65.49,3.96,6.11$. & $65.78,3.80,6.24$. \\
\hline $\mathbf{5}$ & $\mathrm{C}_{26} \mathrm{H}_{17} \mathrm{~F}_{3} \mathrm{~N}_{2} \mathrm{O}_{7} \mathrm{~S}_{2}$ & $52.88,2.90,4.74$. & $53.21,3.04,4.93$. \\
\hline $\mathbf{6}$ & $\mathrm{C}_{19} \mathrm{H}_{14} \mathrm{~N}_{2} \mathrm{O}_{3}$ & $71.69,4.43,8.80$. & $71.40,4.56,8.65$. \\
\hline $\mathbf{1 1}$ & $\mathrm{C}_{25} \mathrm{H}_{17} \mathrm{BrN}_{2} \mathrm{O}_{4} \mathrm{~S}$ & $57.59,3.29,5.37$. & $57.30,3.43,5.49$. \\
\hline $\mathbf{1 2}$ & $\mathrm{C}_{19} \mathrm{H}_{13} \mathrm{BrN}_{2} \mathrm{O}_{2}$ & $59.86,3.44,7.35$. & $60.30,3.27,7.52$. \\
\hline $\mathbf{1 3}$ & $\mathrm{C}_{25} \mathrm{H}_{16} \mathrm{~N}_{2} \mathrm{O}_{4} \mathrm{~S}$ & $68.17,3.66,6.37$. & $68.44,3.51,6.47$. \\
\hline $\mathbf{1 4}$ & $\mathrm{C}_{19} \mathrm{H}_{12} \mathrm{~N}_{2} \mathrm{O}_{2}$ & $75.99,4.03,9.33$. & $75.65,4.21,9.52$. \\
\hline $\mathbf{1 5}$ & $\mathrm{C}_{32} \mathrm{H}_{23} \mathrm{BrN}_{2} \mathrm{O}_{5} \mathrm{~S}$ & $61.25,3.69,4.46$. & $61.54,3.82,4.59$. \\
\hline $\mathbf{1 6}$ & $\mathrm{C}_{26} \mathrm{H}_{19} \mathrm{BrN}_{2} \mathrm{O}_{3}$ & $64.08,3.93,5.75$. & $63.73,4.08,5.87$. \\
\hline $\mathbf{1 7}$ & $\mathrm{C}_{26} \mathrm{H}_{18} \mathrm{~N}_{2} \mathrm{O}_{3}$ & $76.83,4.46,6.89$. & $77.12,4.63,6.75$. \\
\hline $\mathbf{1 8}$ & $\mathrm{C}_{19} \mathrm{H}_{12} \mathrm{~N}_{2} \mathrm{O}_{3}$ & $72.15,3.82,8.86$. & $72.46,3.69,8.99$. \\
\hline $\mathbf{1 9}$ & $\mathrm{C}_{18} \mathrm{H}_{9} \mathrm{NO}_{3}:$ & $75.26,3.16,4.88$. & $74.86,3.30,4.71$. \\
\hline $\mathbf{2 0}$ & $\mathrm{C}_{25} \mathrm{H}_{15} \mathrm{NO}_{4}$ & $76.33,3.84,3.56$. & $76.01,3.97,3.68$. \\
\hline $\mathbf{2 1}$ & $\mathrm{C}_{18} \mathrm{H}_{9} \mathrm{NO}_{4}$ & $71.29,2.99,4.62$. & $71.63,2.84,4.79$. \\
\hline $\mathbf{2 3}$ & $\mathrm{C}_{18} \mathrm{H}_{10} \mathrm{~N}_{2} \mathrm{O}_{3}$ & $71.52,3.33,9.27$. & $71.28,3.50,9.13$. \\
\hline $\mathbf{2 4}$ & $\mathrm{C}_{22} \mathrm{H}_{19} \mathrm{~N}_{3} \mathrm{O}_{2}$ & $73.93,5.36,11.76$ & $74.27,5.18,11.83$. \\
\hline $\mathbf{2 5}$ & $\mathrm{C}_{22} \mathrm{H}_{19} \mathrm{~N}_{3} \mathrm{O}_{3}$ & $70.76,5.13,11.25$. & $71.03,5.02,11.09$. \\
\hline $\mathbf{2 6}$ & $\mathrm{C}_{21} \mathrm{H}_{16} \mathrm{~N}_{2} \mathrm{O}_{4}$ & $69.99,4.48,7.77$. & $70.33,4.35,7.86$. \\
\hline $\mathbf{2 7}$ & $\mathrm{C}_{21} \mathrm{H}_{16} \mathrm{~N}_{2} \mathrm{O}_{5}$ & $67.02,4.28,7.44$. & $69.79,4.45,7.50$. \\
\hline $\mathbf{2 8}$ & $\mathrm{C}_{23} \mathrm{H}_{16} \mathrm{~N}_{4} \mathrm{O}_{2}$ & $72.62,4.24,14.73$. & $72.96,4.10,14.87$. \\
\hline $\mathbf{2 9}$ & $\mathrm{C}_{23} \mathrm{H}_{16} \mathrm{~N}_{4} \mathrm{O}_{3}$ & $69.69,4.07,14.13$. & \\
\hline
\end{tabular}


Routine spectroscopic data

3-(1-Benzenesulfonyl-1H-indol-3-yl)-4-(2-methoxy-phenyl)-1-methyl-pyrrole-2,5-dione (3). Rf (petroleum ether/EtOAc 7/3) 0.86 ; IR $\left(\mathrm{KBr}, \mathrm{cm}^{-1}\right) \vee$ 3402, 2918, 1752, 1684, 1594, 1386, 1168, 734;

${ }^{13} \mathrm{C}$ NMR (DMSO, $\left.62.5 \mathrm{MHz}\right): \delta 26.6\left(\mathrm{CH}_{3}\right), 56.2\left(\mathrm{CH}_{3}\right), 113.8(\mathrm{CH}), 115.3(\mathrm{CH}), 115.5(\mathrm{Cq}), 120.7$ $(\mathrm{Cq}), 122.5(\mathrm{CH}), 123.2(\mathrm{CH}), 125.6(\mathrm{CH}), 127.6(\mathrm{CH}), 129.3(2 \mathrm{CH}), 129.8(\mathrm{Cq}), 130.1(\mathrm{CH}), 132.4$ $(2 \mathrm{CH}), 132.8(\mathrm{Cq}), 133.5(\mathrm{CH}), 133.8(\mathrm{CH}), 134.9(\mathrm{Cq}), 136.2(\mathrm{Cq}), 137.4(\mathrm{Cq}), 138.8(\mathrm{CH}), 159.6$ (Cq), $172.4(\mathrm{CO}), 172.7(\mathrm{CO})$.

3-(1-Benzenesulfonyl-1H-indol-3-yl)-4-(2-hydroxy-phenyl)-1-methyl-pyrrole-2,5-dione (4). Rf (petroleum ether /EtOAc 3/7) 0.55; IR $\left(\mathrm{KBr}, \mathrm{cm}^{-1}\right) \vee 3426,2946,1696,1430,1174,752 ;{ }^{13} \mathrm{C}$ NMR (DMSO, $62.5 \mathrm{MHz}): \delta 27.2\left(\mathrm{CH}_{3}\right), 115.5(\mathrm{CH}), 116.0(\mathrm{Cq}), 118.8(\mathrm{Cq}), 119.8(\mathrm{CH}), 121.8(\mathrm{CH}), 124.6$ $(\mathrm{CH}), 126.5(\mathrm{CH}), 128.2(\mathrm{CH}), 129.8(2 \mathrm{CH}), 130.9(\mathrm{Cq}), 131.3(\mathrm{CH}), 133.1(2 \mathrm{CH}+\mathrm{Cq}), 134.0(\mathrm{Cq})$, $134.1(\mathrm{CH}), 136.9(\mathrm{Cq}), 137.4(\mathrm{Cq}), 138.1(\mathrm{Cq}), 139.5(\mathrm{CH}), 158.8(\mathrm{Cq}), 173.3(\mathrm{CO}), 173.4(\mathrm{CO})$.

\section{3-(1-Benzenesulfonyl-1H-indol-3-yl)-4-(2-trifluoromethylsulfonate-phenyl)-1-methyl-pyrrole-}

2,5-dione (5). Rf (petroleum ether /EtOAc 7/3) 0.35 ; IR $\left(\mathrm{KBr}, \mathrm{cm}^{-1}\right) \vee$ 3156, 2962, 1772, 1708, 1448, 1212, 750, $595 ;{ }^{13} \mathrm{C}$ NMR (DMSO, $\left.62.5 \mathrm{MHz}\right): \delta 24.6\left(\mathrm{CH}_{3}\right), 62.5(\mathrm{Cq}), 111.0(\mathrm{CH}), 113.5(\mathrm{CH})$, $120.5(\mathrm{Cq}), 121.0(\mathrm{CH}), 122.3(\mathrm{Cq}), 123.5(\mathrm{CH}), 123.8(\mathrm{Cq}), 125.6(\mathrm{Cq}), 125.8(\mathrm{Cq}), 126.9(\mathrm{Cq}), 127.0$ $(2 \mathrm{CH}), 129.4(\mathrm{CH}), 129.6(\mathrm{CH}), 130.3(2 \mathrm{CH}), 132.5(\mathrm{CH}), 132.9(\mathrm{CH}), 134.1(\mathrm{CH}), 135.4(\mathrm{Cq}), 136.5$ $(\mathrm{CH}), 146.9(\mathrm{Cq}), 169.4(\mathrm{CO}), 169.5(\mathrm{CO})$.

3-(2-Hydroxy-phenyl)-4-(1H-indol-3-yl)-1-methyl-pyrrole-2,5-dione (6). Rf (petroleum ether /EtOAc 3/7) $0.28 ; \mathrm{IR}\left(\mathrm{KBr}, \mathrm{cm}^{-1}\right) \vee 3556,3105,2952,1724,1703,1452,1386,1176,994 ;{ }^{13} \mathrm{C} \mathrm{NMR}$ (DMSO, 62.5 MHz) : $\delta 24.1\left(\mathrm{CH}_{3}\right), 112.5(\mathrm{CH}), 116.5(\mathrm{Cq}), 121.5(\mathrm{CH}), 123.5(\mathrm{CH}), 124.7(\mathrm{CH}), 126.1$ 
$(\mathrm{Cq}), 127.6(\mathrm{Cq}), 125.5(\mathrm{CH}), 127.2(2 \mathrm{CH}), 128.3(\mathrm{CH}), 128.8(\mathrm{CH}), 130.5(\mathrm{Cq}), 136.9(\mathrm{Cq}), 141.5$ $(\mathrm{CH}), 164.8(\mathrm{Cq}), 172.6(\mathrm{CO}), 172.7(\mathrm{CO})$.

3-(1-Benzenesulfonyl-1H-indol-3-yl)-4-(2-bromo-phenyl)-1-methyl-pyrrole-2,5-dione (11). Rf (petroleum ether /EtOAc 8/2) 0.68 ; IR $\left(\right.$ ATR, $\left.\mathrm{cm}^{-1}\right) \vee 3119,2927,2846,1696,1441,1374,1137,738$; ${ }^{13} \mathrm{C}$ NMR (DMSO, 62.5 MHz) : $\delta 26.0\left(\mathrm{CH}_{3}\right), 113.7(\mathrm{CH}), 115.2(\mathrm{Cq}), 123.0(\mathrm{CH}), 124.8(\mathrm{Cq}), 125.7$ $(\mathrm{CH}), 127.5(\mathrm{CH}), 128.8(2 \mathrm{CH}), 129.3(\mathrm{CH}), 129.7(\mathrm{Cq}), 131.3(\mathrm{CH}), 131.9(2 \mathrm{CH}), 132.7(\mathrm{Cq}), 133.4$ $(\mathrm{CH}), 133.9(\mathrm{CH}), 134.3(\mathrm{Cq}), 134.7(\mathrm{Cq}), 135.9(\mathrm{Cq}), 137.0(\mathrm{CH}), 137.2(\mathrm{Cq}), 138.2(\mathrm{Cq}), 171.3(\mathrm{CO})$, $171.5(\mathrm{CO})$.

3-(2-Bromo-phenyl)-4-(1H-indol-3-yl)-1-methyl-pyrrole-2,5-dione (12). Rf (petroleum ether /EtOAc 8/2) 0.18 ; IR (ATR, $\left.\mathrm{cm}^{-1}\right) \vee 3343,2956,2919,2850,1684,1627,1413,1374,1093,746 ;{ }^{13} \mathrm{C}$ NMR (DMSO, 62.5 MHz) : $\delta 24.7\left(\mathrm{CH}_{3}\right), 105.8(\mathrm{Cq}), 112.9(\mathrm{CH}), 120.8(\mathrm{Cq}), 120.9(\mathrm{CH}), 122.9(\mathrm{CH})$, $124.6(\mathrm{Cq}), 125.3(\mathrm{Cq}), 128.2(\mathrm{CH}), 131.4(\mathrm{CH}), 132.2(\mathrm{Cq}), 132.4(\mathrm{CH}), 132.9(\mathrm{Cq}), 133.3(\mathrm{CH}), 133.4$ $(\mathrm{CH}), 135.4(\mathrm{Cq}), 137.2(\mathrm{Cq}), 170.8(\mathrm{CO}), 171.5(\mathrm{CO})$.

2-(Methyl)-8-(benzenesulfonyl)-benzo[a]pyrrolo[3,4-c]carbazole-1,3(2H,8H)-dione $\quad(13) . \quad \mathrm{Rf}$ (petroleum ether /EtOAc 8/2) 0.38 ; IR $\left(\right.$ ATR, $\left.\mathrm{cm}^{-1}\right) \vee 3121,2932,2856,1703,1461,1401,1183,752$; ${ }^{13} \mathrm{C}$ NMR (DMSO, 62.5 MHz) : $\delta 24.6\left(\mathrm{CH}_{3}\right), 119.5(\mathrm{CH}), 123.5(\mathrm{Cq}), 124.9(\mathrm{CH}), 125.1(\mathrm{CH}), 125.9$ $(\mathrm{CH}), 126.8(\mathrm{Cq}), 127.1(\mathrm{Cq}), 127.2(\mathrm{CH}), 127.3(2 \mathrm{CH}), 127.9(\mathrm{Cq}), 128.0(\mathrm{Cq}), 128.4(\mathrm{CH}), 128.8$ $(\mathrm{CH}), 129.3(\mathrm{Cq}), 129.6(2 \mathrm{CH}), 129.8(\mathrm{CH}), 134.0(\mathrm{CH}), 135.3(\mathrm{Cq}), 141.5(\mathrm{Cq}), 142.1(\mathrm{Cq}), 168.5$ (CO), $169.6(\mathrm{CO})$.

2-(Methyl)-benzo[a]pyrrolo[3,4-c]carbazole-1,3(2H,8H)-dione (14). Rf (petroleum ether /EtOAc 7/3) 0.27 ; IR $\left(A T R, \mathrm{~cm}^{-1}\right)$ v 3323, 2915, 2854, 1680, 1423, 1378, 1072, $734 ;{ }^{13} \mathrm{C}$ NMR (DMSO, 62.5 MHz) : $\delta 24.9\left(\mathrm{CH}_{3}\right), 113.2(\mathrm{CH}), 119.0(\mathrm{Cq}), 122.1(\mathrm{CH}), 122.6(\mathrm{Cq}), 124.1(\mathrm{CH}), 125.3(\mathrm{CH}), 126.1$ 
$(\mathrm{CH}), 127.2(\mathrm{CH}), 127.9(\mathrm{Cq}), 129.0(\mathrm{CH}), 129.5(\mathrm{CH}), 131.4(\mathrm{Cq}), 132.5(\mathrm{Cq}), 133.7(\mathrm{Cq}), 141.3(\mathrm{Cq})$, $141.7(\mathrm{Cq}), 170.5(\mathrm{CO}), 171.4(\mathrm{CO})$.

\section{-(1-Benzenesulfonyl-1H-5-benzyloxyindol-3-yl)-4-(2-bromo-phenyl)-1-methyl-pyrrole-2,5-dione}

(15). Rf (petroleum ether /EtOAc 7/3) 0.77 ; IR (ATR, $\left.\mathrm{cm}^{-1}\right) \vee 3143,2919,1704,1439,1378,1150$, 1089, $754 ;{ }^{13} \mathrm{C}$ NMR (DMSO, 62.5 MHz) : $\delta 25.8\left(\mathrm{CH}_{3}\right), 70.7\left(\mathrm{CH}_{2}\right), 105.6(\mathrm{CH}), 113.3(2 \mathrm{Cq}), 115.7$ $(\mathrm{CH}), 116.8(\mathrm{CH}+\mathrm{Cq}), 124.6(\mathrm{Cq}), 128.2(2 \mathrm{CH}), 128.9(\mathrm{CH}), 129.2(\mathrm{Cq}), 129.3(\mathrm{Cq}), 129.9(2 \mathrm{CH})$, $130.1(\mathrm{Cq}), 131.4(\mathrm{CH}+\mathrm{Cq}), 132.6(\mathrm{CH}), 132.9(\mathrm{Cq}), 133.4(\mathrm{Cq}), 133.6(\mathrm{Cq}), 134.4(\mathrm{CH}), 135.6(\mathrm{Cq})$, $136.5(\mathrm{CH}), 137.7(\mathrm{CH}), 137.8(\mathrm{CH}+\mathrm{Cq}), 156.5(\mathrm{Cq}), 170.9(\mathrm{CO}), 171.7(\mathrm{CO})$.

3-(1H-5-benzyloxyindol-3-yl)-4-(2-bromo-phenyl)-1-methyl-pyrrole-2,5-dione (16). Rf (petroleum ether /EtOAc 7/3) 0.57 ; IR $\left(\mathrm{ATR}, \mathrm{cm}^{-1}\right)$ v 3359, 2919, 2846, 1680, 1619, 1419, 1382, 1064, $732 ;{ }^{13} \mathrm{C}$ NMR (DMSO, 62.5 MHz) : $\delta 24.9\left(\mathrm{CH}_{3}\right), 70.0\left(\mathrm{CH}_{2}\right), 104.1(\mathrm{Cq}), 113.9(\mathrm{CH}), 114.0(\mathrm{Cq}), 124.9(\mathrm{Cq})$, $126.1(\mathrm{Cq}), 127.5(\mathrm{Cq}), 127.7(2 \mathrm{CH}), 128.2(2 \mathrm{CH}), 128.3(\mathrm{Cq}), 128.5(\mathrm{CH}), 129.3(2 \mathrm{CH}), 131.6(\mathrm{Cq})$, $132.4(\mathrm{CH}), 133.1(\mathrm{Cq}), 133.7(\mathrm{CH}), 134.1(\mathrm{Cq}), 135.4(\mathrm{CH}), 137.7(\mathrm{CH}), 153.9(\mathrm{Cq}), 171.0(\mathrm{CO})$, $171.8(\mathrm{CO})$.

2-(Methyl)-5-(benzyloxy)benzo[a]pyrrolo[3,4-c]carbazole-1,3(2H,8H)-dione (17). Rf (petroleum ether /EtOAc 7/3) 0.47 ; IR $\left(\mathrm{ATR}, \mathrm{cm}^{-1}\right)$ v 3347, 3041, 2874, 1676, 1427, 1374, 1296, 1223, $766 ;{ }^{13} \mathrm{C}$ NMR (DMSO, 62.5 MHz) : $\delta 24.2\left(\mathrm{CH}_{3}\right), 71.2\left(\mathrm{CH}_{2}\right), 108.9(\mathrm{CH}), 112.8(\mathrm{Cq}), 117.7(\mathrm{CH}), 118.2(\mathrm{Cq})$, $122.7(\mathrm{Cq}), 123.4(\mathrm{CH}+\mathrm{Cq}), 125.6(\mathrm{CH}), 126.7(\mathrm{Cq}), 128.1(\mathrm{Cq}), 128.5(3 \mathrm{CH}), 128.7(\mathrm{CH}+\mathrm{Cq}), 129.1$ (3CH), $125.9(\mathrm{Cq}), 135.9(\mathrm{Cq}), 141.7(\mathrm{CH}), 154.2(\mathrm{Cq}), 169.9(\mathrm{CO}), 170.7(\mathrm{CO})$.

2-(Methyl)-5-(hydroxy)benzo[a]pyrrolo[3,4-c]carbazole-1,3(2H,8H)-dione (18). Rf (petroleum ether /EtOAc 5/5) 0.32 ; IR (ATR, cm $\left.{ }^{-1}\right) \vee 3347,1676,1427,1328,1190,799,746 ;{ }^{13} \mathrm{C}$ NMR (DMSO, $62.5 \mathrm{MHz}): \delta 23.2\left(\mathrm{CH}_{3}\right), 108.4(\mathrm{CH}), 111.5(\mathrm{Cq}), 112.0(\mathrm{CH}), 116.1(\mathrm{Cq}), 111.5(\mathrm{CH}), 121.9(\mathrm{CH})$, 
$122.1(\mathrm{Cq}), 122.4(\mathrm{Cq}), 124.5(\mathrm{CH}), 125.5(\mathrm{Cq}), 127.1(\mathrm{CH}), 127.6(\mathrm{Cq}), 133.8(\mathrm{CH}+\mathrm{Cq}), 140.5(\mathrm{Cq})$, $151.8(\mathrm{Cq}), 168.9(\mathrm{CO}), 169.8(\mathrm{CO})$.

$5 H$-Furo[3,4-c]benzo[2,3-a]pyrrolo[3,4-c $]$ carbazole-1,3(8H)-dione (19). Rf (petroleum ether /EtOAc 4/6) $0.15 ;$ IR $\left(\right.$ ATR, $\left.\mathrm{cm}^{-1}\right) \vee 3221,3054,2960,1708,1366,1170,1097,154,722 ;{ }^{13} \mathrm{C}$ NMR (DMSO, $62.5 \mathrm{MHz}): \delta 112.5(\mathrm{Cq}), 113.1(\mathrm{CH}), 118.5(\mathrm{Cq}), 121.7(\mathrm{Cq}), 122.2(\mathrm{CH}),, 123.9(\mathrm{CH})$, $124.1(\mathrm{CH}), 124.2(\mathrm{Cq}), 125.6(\mathrm{Cq}), 126.7(\mathrm{Cq}), 127.6(\mathrm{CH}), 128.2(\mathrm{CH}), 127.9(\mathrm{CH}), 129.9(2 \mathrm{Cq}+\mathrm{CH})$, $165.1(\mathrm{CO}), 165.2(\mathrm{CO})$.

\section{5-Benzyloxy-5H-furo[3,4-c]benzo[2,3-a]pyrrolo[3,4-c]carbazole-1,3(8H)-dione $\quad(20) . \quad \mathrm{Rf}$} (petroleum ether /EtOAc 7/3) 0.27 ; IR $\left(\right.$ ATR, $\left.\mathrm{cm}^{-1}\right) \vee 3306,2935,2858,1725,1313,1186,1019,775$; ${ }^{13} \mathrm{C}$ NMR (DMSO, $\left.62.5 \mathrm{MHz}\right): \delta 70.9\left(\mathrm{CH}_{2}\right), 107.7(\mathrm{CH}), 112.8(\mathrm{Cq}), 113.6(\mathrm{CH}), 117.9(\mathrm{CH}), 122.5$ $(\mathrm{Cq}), 123.7(\mathrm{Cq}+\mathrm{CH}), 124.5(\mathrm{Cq}), 125.6(\mathrm{CH}), 126.8(\mathrm{Cq}), 128.6(2 \mathrm{CH}+\mathrm{Cq}), 129.2(\mathrm{Cq}), 129.5(2 \mathrm{CH})$, $129.6(\mathrm{CH}), 129.9(\mathrm{Cq}), 136.1(\mathrm{Cq}), 138.6(\mathrm{Cq}), 142.9(\mathrm{Cq}), 154.9(\mathrm{Cq}), 165.2(\mathrm{CO}), 165.3(\mathrm{CO})$.

5-Hydroxy-5H-furo[3,4-c]benzo[2,3-a]pyrrolo[3,4-c]carbazole-1,3(8H)-dione (21). Rf (petroleum ether /EtOAc 3/7) $0.24 ;$ IR $\left(\right.$ ATR, $\left.\mathrm{cm}^{-1}\right) \vee 3321,3229,1733,1688,1460,1333,1243,738 ;{ }^{13} \mathrm{C} \mathrm{NMR}$ (DMSO, 62.5 MHz) : $\delta 110.3(\mathrm{CH}), 114.4(\mathrm{Cq}), 115.5(\mathrm{CH}), 119.5(\mathrm{Cq}), 119.7(\mathrm{CH}), 124.5(\mathrm{Cq}), 125.7$ $(\mathrm{CH}), 126.0(\mathrm{Cq}), 127.6(\mathrm{CH}), 128.4(\mathrm{CH}), 130.4(\mathrm{Cq}), 131.6(\mathrm{Cq}), 132.8(\mathrm{CH}), 136.7(\mathrm{Cq}), 144.4(\mathrm{Cq})$, $155.3(\mathrm{Cq}), 167.1(2 \mathrm{CO})$.

5-Hydroxy-benzo $[a]$ pyrrolo[3,4-c]carbazole-1,3(2H,8H)-dione (23). Rf (petroleum ether /EtOAc 7/3) 0.47 ; IR $\left(\right.$ ATR, $\left.\mathrm{cm}^{-1}\right) \vee 3349,3327,1726,1447,1337,1182,771 ;{ }^{13} \mathrm{C}$ NMR (DMSO, $\left.62.5 \mathrm{MHz}\right)$ : $\delta 109.3(\mathrm{CH}), 112.5(\mathrm{Cq}), 113.1(\mathrm{CH}), 117.2(\mathrm{CH}), 118.5(\mathrm{Cq}), 122.9(\mathrm{Cq}), 123.5(\mathrm{CH}), 125.7(\mathrm{CH})$, $126.3(\mathrm{Cq}), 126.8(\mathrm{Cq}), 128.4(\mathrm{CH}), 128.8(\mathrm{CH}), 129.6(\mathrm{Cq}), 134.7(\mathrm{Cq}), 141.6(\mathrm{Cq}), 152.8(\mathrm{Cq}), 171.5$ (CO), $172.4(\mathrm{CO})$. 
2-(2-Dimethylaminoethyl)benzo[a]pyrrolo[3,4-c]carbazole-1,3(2H,8H)-dione (24). Rf (petroleum ether /EtOAc 5/5) 0.14 ; IR (ATR, $\mathrm{cm}^{-1}$ ) v 2919, 2838, 1688, 1460, 1374, 1243, 811, $738{ }^{13} \mathrm{C}$ NMR (DMSO, 62.5 MHz) : $\delta 38.05\left(\mathrm{CH}_{2}\right), 47.95\left(2 \mathrm{CH}_{3}\right), 59.63\left(\mathrm{CH}_{2}\right), 114.6(\mathrm{CH}), 120.1(\mathrm{Cq}), 123.5(\mathrm{CH})$, $123.9(\mathrm{Cq}), 125.2(\mathrm{Cq}), 125.5(\mathrm{CH}), 126.7(\mathrm{CH}), 127.5(\mathrm{CH}), 128.2(\mathrm{CH}), 128.6(\mathrm{Cq}), 129.3(\mathrm{Cq}), 130.2$ $(\mathrm{CH}), 130.4(\mathrm{Cq}), 130.9(\mathrm{CH}), 142.6(\mathrm{Cq}), 143.1(\mathrm{Cq}), 171.7(\mathrm{CO}), 172.6(\mathrm{CO})$

2-(2-Dimethylaminoethyl)benzo[a]pyrrolo[3,4-c] carbazole-1,3(2H,8H)-dione (25). Rf (petroleum ether /EtOAc 5/5) 0.17 ; IR (ATR, $\left.\mathrm{cm}^{-1}\right)$ v 3274, 2939, 2833, 2780, 1696, 1468, 1390, 1256, 995, 771 ; ${ }^{13} \mathrm{C}$ NMR (DMSO, 62.5 MHz) : $\delta 36.15\left(\mathrm{CH}_{2}\right), 46.0\left(2 \mathrm{CH}_{3}\right), 57.7\left(\mathrm{CH}_{2}\right), 109.3(\mathrm{CH}), 112.7(\mathrm{Cq}), 113.2$ $(\mathrm{CH}), 117.4(\mathrm{CH}), 122.9(\mathrm{Cq}), 123.4(\mathrm{CH}), 123.6(\mathrm{Cq}), 125.7(\mathrm{CH}), 126.7(\mathrm{Cq}), 128.5(\mathrm{CH}), 128.6(\mathrm{Cq})$, $129(\mathrm{CH}), 134.9(\mathrm{Cq}), 136.2(\mathrm{Cq}), 141.7(\mathrm{Cq}), 153.0(\mathrm{Cq}), 170.0(\mathrm{CO}), 170.9(\mathrm{CO})$.

2-[2-Hydroxy-1-(hydroxymethyl)ethyl]benzo[a]pyrrolo[3,4-c]carbazole-1,3(2H,8H)-dione (26). Rf (petroleum ether /EtOAc 7/3) 0.57 ; IR (ATR, $\mathrm{cm}^{-1}$ ) v 3319, 3217, 3066, 1684, 1455, 1341, 1236, $738 ;{ }^{13} \mathrm{C}$ NMR (DMSO, $\left.62.5 \mathrm{MHz}\right): \delta 57.5(\mathrm{CH}), 59.5\left(2 \mathrm{CH}_{2}\right), 112.8(\mathrm{CH}), 112.9(\mathrm{Cq}), 118.6(\mathrm{Cq})$, $121.8(\mathrm{CH}), 122.2(\mathrm{Cq}), 123.6(\mathrm{CH}), 123.9(\mathrm{CH}), 125.1(\mathrm{CH}+\mathrm{Cq}), 125.8(\mathrm{Cq}), 127.0(\mathrm{Cq}), 127.6(\mathrm{CH})$, $128.8(\mathrm{CH}), 129.3(\mathrm{CH}), 141.0(\mathrm{Cq}), 141.4(\mathrm{CH}), 170.7(\mathrm{CO}), 171.6(\mathrm{CO})$.

2-]2-Hydroxy-1-(hydroxymethyl)ethyl]-5-(hydroxy)benzo[a]pyrrolo[3,4-c]carbazole-1,3(2H,8H)dione (27). Rf (petroleum ether /EtOAc 7/3) 0.62 ; IR (ATR, $\mathrm{cm}^{-1}$ ) v 3355, 3253, 2960, 2923, 2850, 1684, 1455, 1366, 1019, 942, $771 ;{ }^{13} \mathrm{C}$ NMR (DMSO, 62.5 MHz) : $\delta 55.6(\mathrm{CH}), 58.2\left(2 \mathrm{CH}_{2}\right), 108.0$ $(\mathrm{CH}), 111.1(\mathrm{CH}), 111.9(\mathrm{Cq}), 115.9(\mathrm{Cq}), 116.0(\mathrm{CH}), 121.5(\mathrm{Cq}), 122.2(\mathrm{Cq}), 122.8(\mathrm{Cq}), 124.2(\mathrm{CH})$, $125.4(\mathrm{CH}), 126.9(\mathrm{CH}), 127.6(\mathrm{Cq}), 127.7(\mathrm{CH}), 133.6(\mathrm{Cq}), 140.4(\mathrm{Cq}), 151.6(\mathrm{Cq}), 169.3(\mathrm{CO}), 170.2$ (CO).

2-(2-(1H-Imidazol-4-yl)-ethyl)-5-(hydroxy)benzo[a]pyrrolo[3,4-c ]carbazole-1,3(2H,8H)-dione (28). Rf (petroleum ether /EtOAc 5/5) 0.17 ; IR (ATR, $\mathrm{cm}^{-1}$ ) v 3551, 3319, 2923, 2850, 1688, 1460, 
1362, 1239, 1023, $742 ;{ }^{13} \mathrm{C}$ NMR (DMSO, $\left.62.5 \mathrm{MHz}\right): \delta 29.75\left(\mathrm{CH}_{2}\right), 34.8\left(\mathrm{CH}_{2}\right), 110.8(\mathrm{CH}), 116.5$

$(\mathrm{Cq}), 118.7(\mathrm{CH}), 119.9(\mathrm{Cq}), 120.2(\mathrm{CH}), 121.4(\mathrm{CH}), 121.8(2 \mathrm{Cq}), 122.9(\mathrm{CH}), 123.8(\mathrm{Cq}), 124.9$ $(\mathrm{CH}), 125.6(\mathrm{Cq}), 126.6(\mathrm{Cq}), 126.7(\mathrm{CH}), 127.2(\mathrm{CH}), 133.9(\mathrm{CH}), 138.9(\mathrm{CH}), 139.4(\mathrm{Cq}), 161.3(\mathrm{Cq})$, $167.9(\mathrm{CO}), 168.8(\mathrm{CO})$; MS (IS) : $381(\mathrm{M}+1)^{+}$; Anal. calcd for $\mathrm{C}_{23} \mathrm{H}_{16} \mathrm{~N}_{4} \mathrm{O}_{2}: \mathrm{C}, 72.62 ; \mathrm{H}, 4.24 ; \mathrm{N}$, 14.73. Found: C, $72.96 ; \mathrm{H}, 4.10 ; \mathrm{N}, 14.88$.

\section{2-(2-(1H-Imidazol-4-yl)-ethyl)benzo[a]pyrrolo[3,4-c]carbazole-1,3(2H,8H)-dione $\quad(29) . \quad$ Rf} (petroleum ether /EtOAc 5/5) 0.17 ; IR $\left(\right.$ ATR, $\left.\mathrm{cm}^{-1}\right) \vee$ 3355, 2827, 2842, 1684, 1476, 1376, 1190, 795 ; ${ }^{13} \mathrm{C}$ NMR (DMSO, $\left.62.5 \mathrm{MHz}\right): \delta 39.2\left(\mathrm{CH}_{2}\right), 43.5\left(\mathrm{CH}_{2}\right), 106.9(\mathrm{CH}), 110.2(\mathrm{Cq}), 110.7(\mathrm{CH}), 114.8$ $(\mathrm{Cq}), 115.1(\mathrm{CH}), 120.5(\mathrm{CH}), 120.9(\mathrm{Cq}), 121.1(\mathrm{Cq}), 123.2(\mathrm{CH}), 124.3(\mathrm{Cq}), 126.0(2 \mathrm{CH}), 126.3$ (Cq), $126.5(\mathrm{CH}), 132.4(\mathrm{Cq}), 138.8(\mathrm{CH}), 139.2(\mathrm{Cq}), 139.3(\mathrm{Cq}), 150.5(\mathrm{Cq}), 167.5(\mathrm{CO}), 168.4(\mathrm{CO})$ ; MS (IS) : $397(\mathrm{M}+1)^{+}$; Anal. calcd for $\mathrm{C}_{23} \mathrm{H}_{16} \mathrm{~N}_{4} \mathrm{O}_{3}$ : C, 69.69; H, 4.07; N, 14.13. Found: C, $69.31 ; \mathrm{H}$, $4.23 ; \mathrm{N}, 14.04$. 